\title{
HYPERBOLIC MANIFOLDS AND PSEUDO-ARITHMETICITY
}

\author{
VINCENT EMERY AND OLIVIER MILA
}

In memoriam E. B. Vinberg

\begin{abstract}
We introduce and motivate a notion of pseudo-arithmeticity, which possibly applies to all lattices in $\mathrm{PO}(n, 1)$ with $n>3$. We further show that under an additional assumption (satisfied in all known cases), the covolumes of these lattices correspond to rational linear combinations of special values of $L$-functions.
\end{abstract}

\section{INTRODUCTION}

The study of hyperbolic 3-manifolds of finite volume has many relations with number theory, with a central role in this context being played by the notion of the invariant trace field (see [17]). The work of Vinberg [31] allows to define a comparable invariant (the adjoint trace field) for any locally symmetric space. This paper studies finite volume hyperbolic manifolds of dimensions $n>3$ from the perspective of the adjoint trace field, and the algebraic groups that are naturally associated with them (see Theorem 1.2).

Our main purpose is to introduce and motivate a notion of pseudo-arithmeticity, which to the best of our knowledge applies to all currently known lattices in $\operatorname{PO}(n, 1)$ with $n>3$. In this introduction we state two main results: Theorem 1.5, where we show that the classical hyperbolic manifolds obtained by gluing are pseudoarithmetic; and Theorem 1.12 in which it is proved that under some mild assumption (defined in Sect. 1.5) the covolumes of pseudo-arithmetic lattices correspond to rational linear combinations of covolumes of arithmetic lattices.

1.1. Hyperbolic isometries and algebraic groups. We denote by $\mathbf{H}^{n}$ the hyperbolic $n$-space, and we identify its group of isometries with the Lie group $\mathrm{PO}(n, 1)$. Let $\mathbf{P O}$ denote the real algebraic group such that $\mathbf{P O}(\mathbb{R})=\mathrm{PO}(n, 1)$, and $\mathbf{P O}^{+}$ its identity component. Note the following dichotomy:

- for $n$ even $\mathbf{P O}$ is connected, so that $\mathbf{P O} \mathbf{O}^{+}(\mathbb{R})=\mathrm{PO}(n, 1)$;

- for $n$ odd $\mathbf{P O}$ has two connected components, and we have $\mathbf{P O}^{+}(\mathbb{R})=$ $\mathrm{PO}(n, 1)^{\circ}$.

Recall that any complete hyperbolic $n$-manifold $M$ can be written as a quotient $M=\Gamma \backslash \mathbf{H}^{n}$, where $\Gamma \subset \mathrm{PO}(n, 1)$ is a torsion-free discrete subgroup (uniquely determined up to conjugacy). The manifold $M$ has finite volume exactly when $\Gamma$ is a lattice in $\mathrm{PO}(n, 1)$. We will usually assume that $M$ is orientable, i.e., $\Gamma \subset \mathrm{PO}(n, 1)^{\circ}$. Then by Borel's density theorem $\Gamma$ is Zariski-dense in $\mathbf{P O}^{+}$.

Received by the editors May 16, 2019, and, in revised form, November 15, 2019.

2020 Mathematics Subject Classification. Primary 22E40; Secondary 20G30, 51M25.

This work was supported by the Swiss National Science Foundation, Project number PP00P2_157583.

(C)2021 by the authors under Creative Commons Attribution-Noncommercial 3.0 License (CC BY NC 3.0) 
Let $K / k$ be a field extension; for an algebraic $k$-group $\mathbf{G}$ the symbol $\mathbf{G}_{K}$ denotes the $K$-group obtained by scalar extension (base change induced by $k \subset K$ ). In this case $\mathbf{G}$ is said to be a $k$-form of $\mathbf{G}_{K}$. Assume that $k$ is a number field, with ring of integers $\mathcal{O}_{k}$. Then a $k$-form $\mathbf{G}$ of $\mathbf{P O}^{+}$(or of $\mathbf{P O}$ ) is called admissible (for $\mathrm{PO}(n, 1))$ if the Lie group $\mathbf{G}\left(k \otimes_{\mathbb{Q}} \mathbb{R}\right)^{\circ}$ contains exactly one noncompact factor, isomorphic to $\mathrm{PO}(n, 1)^{\circ}$. In this case $\mathbf{G}\left(\mathcal{O}_{k}\right)$ is a lattice in $\operatorname{PO}(n, 1)$.

Remark 1.1. For $n>3$ the admissibility condition implies that $k$ is totally real.

1.2. Trace fields and ambient groups. For any subgroup $\Gamma \subset \mathrm{PO}(n, 1)$ with $n>3$, we define its (adjoint) trace field as the subfield of $\mathbb{R}$ given by

$$
k=\mathbb{Q}(\{\operatorname{tr}(\operatorname{Ad} \gamma) \mid \gamma \in \Gamma)\}),
$$

where $\mathrm{Ad}: \operatorname{PO}(n, 1) \rightarrow \mathrm{GL}(\mathfrak{g})$ is the adjoint representation. In case $\Gamma$ is a lattice, it follows from Weil's local rigidity that $k$ is a number field (see [23, Prop. 1.6.5]). The work of Vinberg [31] shows the following.

Theorem 1.2 (Vinberg). Let $\Gamma$ be a Zariski-dense subgroup of $\mathbf{P O}^{+}(\mathbb{R})$ (resp. of $\mathbf{P O}(\mathbb{R}))$, with trace field $k$. Then

(1) $k$ is an invariant of the commensurability class of $\Gamma$;

(2) there exists a $k$-form $\mathbf{G}$ of $\mathbf{P O} \mathbf{O}^{+}$(resp. of $\mathbf{P O}$ ) such that $\Gamma \subset \mathbf{G}(k)$.

Since $\Gamma$ is Zariski-dense, the group $\mathbf{G}$ is uniquely determined by $\Gamma$ (up to $k$ isomorphism), and it is a commensurability invariant. We call it the ambient group of $\Gamma$. If $\mathbf{G}$ is admissible then $\Gamma$ is called quasi-arithmetic. If moreover $\Gamma$ is commensurable with $\mathbf{G}\left(\mathcal{O}_{k}\right)$, it is called arithmetic. We use the same terminology for the corresponding quotient $\Gamma \backslash \mathbf{H}^{n}$.

Remark 1.3. This definition of arithmetic lattices differs from the usual one (see for instance [23, Sect. 3.6]); but it is equivalent to it. The equivalence essentially follows from the fact that trace fields of arithmetic lattices coincide with their fields of definition (see [26, Lemma 2.6]).

Remark 1.4. For $n=3$ the group $\mathrm{PO}(3,1)^{\circ}$ is isomorphic to $G=\mathrm{PGL}_{2}(\mathbb{C})$ and thus carries a complex structure. Taking the complex adjoint representation $G \rightarrow$ $\mathrm{GL}\left(\mathfrak{g}_{\mathbb{C}}\right)$ instead in the definition, the adjoint trace field coincides with the "invariant trace field" (see [17, Exercise $3.3(4)]$ ).

1.3. The case of glued manifolds. To date for $n>3$ there are two sources of nonarithmetic lattices in $\mathrm{PO}(n, 1)$ :

(1) Hyperbolic reflection groups 1 some of which can be proved to be nonarithmetic by using Vinberg's criterion 30 .

(2) Hyperbolic manifolds constructed by gluing together "pieces" of arithmetic manifolds along pairwise isometric totally geodesic hypersurfaces.

There are several constructions that fit the description in (2) (see [2,9,27]), all of which can be thought as (clever) variations of the original method 2 by Gromov and Piateski-Shapiro [10. Their building blocks are always arithmetic pieces $M_{i}$,

\footnotetext{
${ }^{1}$ Note that hyperbolic reflections groups (of finite covolume) cannot exist for $n \geq 996$, and no examples are known for $n>21$ (see [1, Sect. 1]).

${ }^{2}$ Note that this method has also inspired Vinberg's recent paper [32, where "hybrid" nonarithmetic reflection groups are constructed. This shows in particular that the two sources (1) and (2) are not disjoint.
} 
i.e., hyperbolic $n$-manifolds with totally geodesic boundary taken inside arithmetic manifolds $\Gamma_{i} \backslash \mathbf{H}^{n}$ (see Sect. 2.4 2.5) . We will see in Corollary 2.6 that two such pieces $M_{i}$ and $M_{j}$ cannot be glued together unless $\Gamma_{i}$ and $\Gamma_{j}$ have the same field of definition (equivalently, the same trace field).

The following theorem applies to all nonarithmetic lattices constructed in the sense of (2). By a multiquadratic extension $K / k$ we mean a (possibly trivial) field extension of the form $K=k\left(\sqrt{a_{1}}, \ldots, \sqrt{a_{s}}\right)\left(a_{i} \in k, s \geq 0\right)$.

Theorem 1.5. For $n>3$, let $M=\Gamma \backslash \mathbf{H}^{n}$ be a hyperbolic $n$-manifold obtained by gluing together a finite number of arithmetic pieces $M_{i} \subset \Gamma_{i} \backslash \mathbf{H}^{n}$, each of the arithmetic lattices $\Gamma_{i}$ being defined over $k$. Then:

(1) The trace field $K$ of $\Gamma$ is a totally real multiquadratic extension of $k$.

(2) The ambient group of $\Gamma$ is $\mathbf{G}_{K}$, where $\mathbf{G}$ is the ambient group of any of the arithmetic lattices $\Gamma_{i}$.

The exact description of the trace field $K$ - and in particular the degree of $K / k-$ depends on the pieces $M_{i}$ and the way they are glued together. A precise treatment is the subject of a separate article by the second author [20]. See also [21].

1.4. Pseudo-arithmetic lattices. Theorem 1.5 motivates the following definitions:

Definition 1.6 (Pseudo-admissible). We call pseudo-admissible (over $K / k$ ) an algebraic group of the form $\mathbf{G}_{K}$, where $K / k$ is a finite (possibly trivial) totally real multiquadratic extension and $\mathbf{G}$ is an admissible $k$-group.

Definition 1.7 (Pseudo-arithmetic). Let $n>3$. A lattice $\Gamma \subset \mathrm{PO}(n, 1)$ will be called pseudo-arithmetic if its ambient group is pseudo-admissible.

It follows from the definition that (quasi-)arithmetic lattices are pseudo-arithmetic. Theorem 1.5 shows that lattices obtained by gluing arithmetic pieces are pseudo-arithmetic.

In Sect. 4 we will present a method to test if a given reflection group is pseudoarithmetic or not; this can be thought as an extension of Vinberg's (quasi-)arithmeticity criterion [30. We have applied our method on the full list of groups provided with the software CoxIter (see [11]), which contains about a hundred non-quasi-arithmetic Coxeter groups for $n>3$ : all of them turn out to be pseudoarithmetic. A particularly interesting example is the Coxeter group $\Delta_{5}$ presented in Figure 1, it has recently been shown (combining work of Fisher et. al. [8, Sect. 6.2] and of the second author [20]) that $\Delta_{5}$ is not commensurable with any lattice obtained by gluing arithmetic pieces. At this point it is natural to ask:

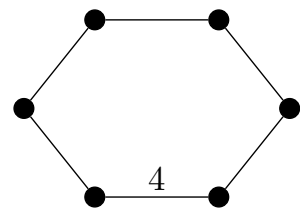

Figure 1. A pseudo-arithmetic Coxeter 5-simplex group: $\Delta_{5}$

Question 1.8. Let $\Gamma \subset \mathrm{PO}(n, 1)$ be a lattice, with $n>3$. Is $\Gamma$ necessarily pseudoarithmetic? 
Remark 1.9. In dimension $n=3$ the abundance of hyperbolic manifolds obtained by Dehn surgeries makes very unlikely a positive answer to an analogue of this question.

Note that by definition the trace field $K$ of a pseudo-arithmetic lattice is totally real. The following question - thus weaker than Question 1.8- seems easier to answer, yet we do not know its current status.

Question 1.10. Let $n>3$. Is the trace field of a lattice $\Gamma \subset \operatorname{PO}(n, 1)$ necessarily totally real?

1.5. First type lattices. A lattice $\Gamma \subset \mathrm{PO}(n, 1)$, is said to be of the first type if its ambient group is of the form $\mathbf{G}=\mathbf{P} \mathbf{O}_{q}$ (resp., $\mathbf{G}=\mathbf{P} \mathbf{O}_{q}^{+}$), where $\mathbf{P} \mathbf{O}_{q}$ denotes the projective orthogonal group (resp., its identity component) of a quadratic form $q$ defined over the trace field $K$; see Sect. 2.1 for details. This extends the terminology sometimes used for arithmetic lattices. For $n$ even, any $K$-form of PO is of the type $\mathbf{P O}_{q}$, and thus for those dimensions all lattices are trivially of the first type. It follows from part (2) of Theorem 1.5 that lattices obtained by gluing arithmetic pieces are of the first type, since such pieces contain totally geodesic hypersurfaces (see Prop. 2.2). Moreover, every Coxeter group is of the first type (the corresponding quadratic form can be read off the Gram matrix, see [31, §4]). To summarize, all currently known lattices in $\mathrm{PO}(n, 1)$ with $n>3$ are either:

- arithmetic, or

- pseudo-arithmetic of the first type.

A lattice simultaneously belongs to both categories if and only if it is arithmetic of the first type (sometimes also called "standard" arithmetic lattices).

In the rest of the paper we will often make implicit use of the following.

Lemma 1.11. Let $\mathbf{G}=\mathbf{P O}_{q}$ be pseudo-admissible over $K / k$, for some quadratic form $q$ over $K$. Then $\mathbf{G} \cong \mathbf{P O}_{f, K}$ for some quadratic form $f$ over $k$.

Proof. By definition $\mathbf{G}=\mathbf{G}_{0, K}$ for some admissible $k$-group $\mathbf{G}_{0}$. Note that the extension $K / k$ is multiquadratic, whence Galois. For any $\sigma \in \operatorname{Gal}(K / k)$, we thus have $\mathbf{P} \mathbf{O}_{\sigma_{q}} \cong \mathbf{P O}_{q}$. This implies that ${ }^{\sigma} q=a_{\sigma} q \circ A_{\sigma}$ for some $a_{\sigma} \in K^{\times}$and $A_{\sigma} \in \mathbf{G L}_{n}(K)$. The maps $\sigma \mapsto a_{\sigma}$ and $\sigma \mapsto A_{\sigma}$ are easily seen to be 1-cocycles, so that $a_{\sigma}=b \sigma\left(b^{-1}\right)$ and $A_{\sigma}=B \sigma\left(B^{-1}\right)$ for some $b \in K^{\times}$and $B \in \mathbf{G L}_{n}(K)$ respectively (Hilbert's Theorem 90). It follows that $f=b q \circ B$ is invariant by $\operatorname{Gal}(K / k)$ and thus defined over $k$, with $\mathbf{G} \cong \mathbf{P O}_{f, K}$.

1.6. Volumes. Let $\mathbf{G}=\mathbf{P} \mathbf{O}_{f, K}\left(\right.$ or $\left.\mathbf{P} \mathbf{O}_{f, K}^{+}\right)$be a pseudo-admissible group over $K / k$. Then we may associate with $\mathbf{G}$ a set of arithmetic lattices in the following way. Let us assume that $K$ is explicitly given by $K=k\left(\sqrt{a_{1}}, \ldots, \sqrt{a_{r}}\right)$, i.e., the degree of $K / k$ is $s=2^{r}$. We will assume (as we may) that the quadratic form $f$ is diagonal in the variables $x_{0}, \ldots, x_{n}$, with negative coefficient in $x_{0}$. For any multiindex $i \in\{0,1\}^{r}$ we set $\alpha_{i}=\sqrt{a_{1}^{i_{1}} \cdots a_{r}^{i_{r}}}$, and $f_{i}=f\left(x_{0}, \ldots, x_{n-1}, \alpha_{i} x_{n}\right)$. All the $a_{i}$ are totally positive since $K$ is totally real by assumption. It follows that all $f_{i}$ are admissible quadratic forms over $k$. For each $i \in\{0,1\}^{r}$ we choose an arithmetic subgroup $\Gamma_{i} \subset \mathbf{P O}_{f_{i}}(k)$, i.e., a subgroup commensurable with $\mathbf{P} \mathbf{O}_{f_{i}}\left(\mathcal{O}_{k}\right)$. We say that the set of arithmetic lattices $\left\{\Gamma_{i} \mid 0 \leq i \leq 2^{r}\right\}$ is subordinated to $\mathbf{G}$. 
We will show that - in some precise sense - the homology over $\mathbb{Q}$ of the group $\mathbf{G}(K)$ is generated by classes associated with the $\Gamma_{i}$; see Theorem 3.5. A direct consequence for the volume is the following.

Theorem 1.12. Let $\Gamma \subset \mathrm{PO}(n, 1)$ be a pseudo-arithmetic lattice of the first type (with $n>3$ ), and $\left\{\Gamma_{i}\right\}$ a set of arithmetic lattices subordinated to the ambient group of $\Gamma$. Then

$$
\operatorname{vol}\left(\Gamma \backslash \mathbf{H}^{n}\right)=\sum_{i} \beta_{i} \operatorname{vol}\left(\Gamma_{i} \backslash \mathbf{H}^{n}\right)
$$

for some $\beta_{i} \in \mathbb{Q}$.

For $n$ even the result readily follows from the Gauss-Bonnet theorem, and Theorem 1.12 has no interest there (but Theorem 3.5 presumably has). For manifolds obtained by gluing the result was already known; see [7, Sect. 1.4]. Note also that for quasi-arithmetic lattices (which corresponds to the case $r=0$ above) the result is proved in [7, Theorem 1.3], and the condition "of the first type" is superfluous.

The covolume of arithmetic lattices is well-understood: it is essentially expressible by means of special values of $L$-functions (see [24,25]). In particular the values of the summands $\operatorname{vol}\left(\Gamma_{i} \backslash \mathbf{H}^{n}\right)$ can be determined up to rationals. The following example illustrates Theorem 1.12 with the case of the Coxeter group $\Delta_{5}$ (which was introduced in Sect. 1.4).

Example 1.13. It was already proved in [30, Sect. III] that the nonuniform Coxeter group $\Delta_{5} \subset \mathrm{PO}(5,1)$ is nonarithmetic, with trace field $K=\mathbb{Q}(\sqrt{2})$. It is the unique hyperbolic simplex group that is nonarithmetic for $n>3$. Applying the procedure described in Sect. 4.1, one finds from its Gram matrix that $\Delta_{5} \subset \mathbf{P O}_{f}(K)$, with $f=f_{0}$ given in (1.3). This shows that $\mathbf{P} \mathbf{O}_{f, K}$ is pseudo-admissible, and thus $\Delta_{5}$ is pseudo-arithmetic of the first type. A subordinated set of arithmetic lattices is given by $\left\{\Gamma_{i}=\mathbf{P O}_{f_{i}}(\mathbb{Z}) \mid i=0,1\right\}$, where

$$
\begin{aligned}
& f_{0}=-x_{0}^{2}+x_{1}^{2}+\cdots+x_{5}^{2} ; \\
& f_{1}=-x_{0}^{2}+x_{1}^{2}+\cdots+2 x_{5}^{2} .
\end{aligned}
$$

We have (see for instance [7, Prop. 2.1] and the references therein):

$$
\begin{aligned}
& \operatorname{vol}\left(\Gamma_{0} \backslash \mathbf{H}^{n}\right) \in \zeta(3) \cdot \mathbb{Q}^{\times} ; \\
& \operatorname{vol}\left(\Gamma_{1} \backslash \mathbf{H}^{n}\right) \in \sqrt{2} L\left(\chi_{8}, 3\right) \cdot \mathbb{Q}^{\times},
\end{aligned}
$$

where $L\left(\chi_{8}, \cdot\right)$ is the Dirichlet $L$-function with nontrivial primitive character mod 8. Equivalently, $L\left(\chi_{8}, s\right)=\zeta_{\mathbb{Q}(\sqrt{2})}(s) / \zeta(s)$; see [34, Sect. 11].

The covolume of $\Delta_{5}$ has been studied by Kellerhals, who obtained a closed formuld 3 in [12, Sect. 3] (where $\Delta_{5}=\widehat{A U}_{5}$ ). Based on this, the following approximation has been found in collaboration with Steve Tschantz. Its accuracy has been checked up to 160 digits.

$$
\begin{aligned}
\operatorname{vol}\left(\Delta_{5} \backslash \mathbf{H}^{5}\right) & \approx \frac{73}{2^{9} \cdot 3^{2} \cdot 5} \zeta(3)+\frac{1}{2^{3} \cdot 3^{2} \cdot 5} \sqrt{2} L\left(\chi_{8}, 3\right) \\
& =0.00757347442200786763497722 \ldots
\end{aligned}
$$

\footnotetext{
${ }^{3}$ Note that in 12 the value on p.350 differs from the one on p.338, and the former is not correct.
} 
Proving a sharp equality in (1.4) seems out of reach with the methods presented in this article. In particular, the method used to prove Theorem 1.12 does not provide any information about the $\beta_{i}$ (or even about their signs).

\subsection{Final remarks.}

1.7.1. The definition of pseudo-arithmeticity can be transferred verbatim for lattices in $\mathrm{PU}(n, 1)(n \geq 2)$. Nonarithmetic lattices in $\mathrm{PU}(n, 1)$ are known to exist for $n=2,3$, and none of the known examples is quasi-arithmetic. In $\mathrm{PU}(2,1)$ there exist nonarithmetic lattices whose trace fields are not quadratic extensions (see [4, Table A.2]); in particular they cannot be pseudo-arithmetic.

1.7.2. In [10, Question 0.4] Gromov and Piateski-Shapiro famously asked whether for any lattice $\Gamma \subset \operatorname{PO}(n, 1)$ of sufficiently large dimension the quotient $\Gamma \backslash \mathbf{H}^{n}$ admits a nice partition into "subarithmetic pieces". In some weak sense (i.e., at the level of homology), Theorem 3.5 gives a positive answer to this question for the class of pseudo-arithmetic lattices of the first type. It is not clear however if a statement closer to their original formulation can be achieved for those lattices; in particular, if a positive answer to the following question could hold:

Question 1.14. Let $\Gamma \subset \mathrm{PO}(n, 1)$ be a pseudo-arithmetic lattice of the first type $(n>3)$, and let $\left\{\Gamma_{i}\right\}$ be a set of arithmetic lattices subordinated to its ambient group. Does the collection of subgroups $\left\{\Gamma \cap \Gamma_{i}\right\}$ generate the group $\Gamma$ ?

\section{Pseudo-ARithmeticity of Gluings}

The goal of this section is to prove Theorem 1.5

2.1. Rational hyperplanes. Let $(V, f)$ be an admissible quadratic space over a number field $k$, i.e., $f: V \rightarrow k$ is an admissible quadratic form. We denote by $\mathbf{O}(V, f)$, or simply $\mathbf{O}_{f}$, the algebraic group of orthogonal transformations of $(V, f)$. We denote by $\mathbf{P O}(V, f)=\mathbf{P O}_{f}$ its adjoint form (it corresponds to the group $\mathbf{P G O}(V, f)$ in the notation of [13]). For a field extension $K / k$, the group of $K$ points $\mathbf{P} \mathbf{O}_{f}(K)$ can be concretely described as the quotient $\mathrm{GO}\left(V_{K}, f\right) / K^{\times}$, where $\mathrm{GO}\left(V_{K}, f\right)$ is the group of similitudes of $V_{K}=V \otimes_{k} K$ (see [13, Sect. 12.A and Sect. 23.B]). In particular, $\mathbf{P O}(V, f)$ acts $k$-rationally on the projective space $\mathbf{P} V_{\mathbb{C}}$, and so does its identity component $\mathbf{P O}^{+}(V, f)=\mathbf{P} \mathbf{O}_{f}^{+}$.

The following is a model for the hyperbolic $n$-space $\mathbf{H}^{n}$, with group of isometries $\mathbf{P O}_{f}(\mathbb{R})$ :

$$
\mathbf{H}(V, f)=\left\{x \in \mathbf{P} V_{\mathbb{R}} \mid f(x)<0\right\} .
$$

Any hyperplane in $\mathbf{H}(V, f)$ corresponds to the image of a subspace $W \subset V_{\mathbb{R}}$, with $W=e^{\perp}$ for some $e \in V_{\mathbb{R}}$ such that $f(e)>0$. Such a hyperplane will be denoted by $\mathbf{H}(W) \subset \mathbf{H}(V, f)$. We shall say that $\mathbf{H}(W)$ is $k$-rational if $W$ is, i.e., $W=e^{\perp}$ for some $e \in V$. This is equivalent to saying that the projective space $\mathbf{P} W$ is $k$-closed in $\mathbf{P} V_{\mathbb{R}}$. A useful characterization is the following.

Lemma 2.1. Let $\mathbf{H}(W) \subset \mathbf{H}(V, f)$ be a hyperplane, and let $\rho \in \mathbf{P O}_{f}(\mathbb{R})$ denote the reflection through $\mathbf{H}(W)$. Then $\mathbf{H}(W)$ is k-rational exactly when $\rho \in \mathbf{P O}_{f}(k)$.

Proof. Let $B$ be the bilinear form associated with $f$. If $W=e^{\perp}$ for some $e \in V=$ $V_{k}$, it follows immediately from the formula $\rho(x)=x-\frac{B(x, e)}{B(e, e)} x$ that $\rho$ is $k$-rational. For the converse implication, note that $\mathbf{P} W$ corresponds to the set of fixed points 
of $\rho$, and thus $\rho \in \mathbf{P O}_{f}(k)$ implies that $\mathbf{P} W$ (and thus $\left.\mathbf{H}(W)\right)$ is $k$-rational since the action is defined over $k$.

2.2. Sharp hypersurfaces. Gluings are realized along totally geodesic embedded hypersurfaces of finite volume. To simplify the following discussion, we will call sharp such a hypersurface embedded in a hyperbolic manifold $M$ of finite volume. Note that if $M$ is compact then any totally geodesic hypersurface embedded in $M$ is sharp.

Proposition 2.2. For $n>3$, let $\Gamma \subset \mathrm{PO}(n, 1)^{\circ}$ be an arithmetic lattice over $k$ such that $\Gamma \backslash \mathbf{H}^{n}$ contains a sharp hypersurface $N$. Then

(1) $\Gamma$ is of the first type, i.e., its ambient group is $\mathbf{P O}^{+}(V, f)$ for some admissible quadratic space $(V, f)$ over $k$.

(2) Any hyperplane lift of $N$ in $\mathbf{H}(V, f)$ is k-rational.

Proof. The first statement is already known; see for instance [19, Sect. 10]. For (2) we consider $N \subset \Gamma \backslash \mathbf{H}(V, f)$, with $\Gamma \subset \mathbf{P O}_{f}(k)$. Let $\mathbf{H}(W) \subset \mathbf{H}(V, f)$ be a hyperplane lift of $N$, and let $\rho \in \mathbf{P O}_{f}(\mathbb{R})$ be the associated reflection. Let $\Lambda$ be the stabilizer of $\mathbf{H}(W)$ in $\Gamma$, so that $N=\Lambda \backslash \mathbf{H}(W)$. The latter being sharp, we have that $\Lambda$ is Zariski-dense in the group of isometries of $\mathbf{H}(W)$. It follows that the centralizer $\mathbf{Z}$ of $\Lambda$ in $\mathbf{P O} \mathbf{O}_{f}$ consists of two elements: 1 and $\rho$ (since this holds over $\mathbb{C}$ for the centralizer of $\mathrm{PO}_{n}(\mathbb{C})$ in $\mathrm{PO}_{n+1}(\mathbb{C})$ ). But in a $k$-group of order 2 , the nontrivial element must be a $k$-point (for it is easily seen to be invariant under the Galois group). That is, $\rho \in \mathbf{P} \mathbf{O}_{f}(k)$, and the result follows from Lemma 2.1.

Corollary 2.3. Let $M$ be an arithmetic hyperbolic $n$-manifold defined over $k$, with $n>3$. Then any sharp hypersurface in $M$ is arithmetic of the first type, and defined over $k$.

Proof. Let $M=\Gamma \backslash \mathbf{H}(V, f)$, and $N \subset M$ be a sharp hypersurface, i.e., $N=$ $\Lambda \backslash \mathbf{H}(W)$ for $\Lambda$ the stabilizer in $\Gamma$ of some $k$-rational hyperplane $\mathbf{H}(W)$. The lattice $\Lambda$ is known to be arithmetic; see [19, Theorem 3.2]. Let $f_{0}$ be the restriction of $f$ to $W$. Then $\mathbf{P O}^{+}\left(W, f_{0}\right)$ is a $k$-group admissible for $\mathrm{PO}(n-1,1)$, whose $k$-points contain the arithmetic subgroup $\Lambda$. We conclude that $\mathbf{P O}^{+}\left(W, f_{0}\right)$ is the ambient group of $\Lambda$.

2.3. Extending similitudes. For a similitude $\phi:\left(V_{0}, f_{0}\right) \rightarrow\left(V_{1}, f_{1}\right)$ between quadratic spaces, we denote by $\operatorname{conj}(\phi): \mathbf{P O}\left(V_{0}, f_{0}\right) \rightarrow \mathbf{P O}\left(V_{1}, f_{1}\right)$ the map $g \mapsto$ $\phi \circ g \circ \phi^{-1}$.

Lemma 2.4. Let $\left(V_{0}, f_{0}\right)$ and $\left(V_{1}, f_{1}\right)$ be two nondegenerate quadratic spaces over a field $k$, each of them containing a quadratic subspace $W_{i} \subset V_{i}(i=0,1)$ of codimension 1. Assume that there exists a similitude $\varphi: W_{0} \rightarrow W_{1}$. Then there exists an extension $F / k$ which is at most quadratic such that $\varphi$ extends to a similitude $\phi: V_{0} \otimes_{k} F \rightarrow V_{1} \otimes_{k} F$. In particular, conj $(\phi): \mathbf{P O}\left(V_{0}, f_{0}\right) \rightarrow \mathbf{P O}\left(V_{1}, f_{1}\right)$ is defined over $F$.

Proof. If $V=(V, f)$ is a quadratic space, we write $\lambda V$ for the quadratic space $(V, \lambda f)$. For $i=0,1$, we have a decomposition $V_{i}=W_{i} \perp S_{i}$ where $S_{i}=W_{i}^{\perp}$ is of dimension 1. Thus $S_{0}$ and $S_{1}$ are similar; let $\alpha \in k$ be such that $S_{0}$ is isomorphic to $\alpha S_{1}$. Further let $\lambda \in k$ be such that $\varphi: W_{0} \rightarrow \lambda W_{1}$ is an isomorphism. Then if $F$ denotes the field extension $F=k(\sqrt{\lambda / \alpha})$, we have $S_{0} \otimes_{k} F \cong \lambda S_{1} \otimes_{k} F$. This allows to extend $\varphi$ to an isomorphism $V_{0} \otimes_{k} F \cong \lambda V_{1} \otimes_{k} F$, as desired. 
2.4. Hyperbolic pieces. By a hyperbolic piece (of dimension $n$ ) we mean a complete orientable hyperbolic $n$-manifold of finite volume with a (possibly empty) boundary consisting of finitely many sharp hypersurfaces. We say that a piece is singular if it has nonempty boundary, and regular otherwise (in which case it is just a hyperbolic manifold in the sense of Sect. 1.11). A singular piece $M$ can always be embedded in a regular one of the same dimension: it suffices to consider the "double" $\widehat{M}$ of $M$ obtained by gluing together two copies of $M$ along each boundary component ( $\widehat{M}$ is complete according to [10, 2.10.B]).

Let $M$ be a singular hyperbolic piece. Its universal cover $\widetilde{M}$ is isometric to an infinite intersection of half-spaces in $\mathbf{H}^{n}$; see [18, Sect. 3.5.1]. The fundamental group $\pi_{1} M$ of $M$ identifies with a discrete subgroup $\Gamma \subset \mathbf{P O}^{+}(\mathbb{R})$ stabilizing $\widetilde{M}$ and such that $M \cong \Gamma \backslash \widetilde{M}$. It is clear that any two choices of universal covers for $M$ in $\mathbf{H}^{n}$ are conjugate by an isometry, and thus, up to conjugacy, the discrete subgroup $\Gamma$ is uniquely determined by $M$. The trace field of $M$ is defined as the trace field of $\Gamma$ (see Sect. [1.2). Furthermore, by [10, 1.7.B] we have that $\Gamma$ is Zariskidense in $\mathbf{P O}^{+}(\mathbb{R})$, and using Theorem 1.2 there is therefore an intrinsic notion of ambient group for $\Gamma$.

2.5. Arithmetic pieces. We define an arithmetic piece $M$ (of dimension $n$ ) as a singular hyperbolic piece of dimension $n$ that embeds into an arithmetic hyperbolic manifold $M_{0}=\Gamma_{0} \backslash \mathbf{H}^{n}$. Thus $M=\Gamma \backslash \widetilde{M}$, where $\widetilde{M} \subset \mathbf{H}^{n}$ and $\Gamma=\operatorname{Stab}_{\Gamma_{0}}(\widetilde{M})$ is a subgroup of infinite index in $\Gamma_{0}$ (see [10, 2.10.A]).

Lemma 2.5. Let $M \subset M_{0}$ be an arithmetic piece as above, of dimension $n>3$. Then $M$ and $M_{0}$ have the same trace field and the same ambient group.

Proof. Let $k$ be the field of definition of $M_{0}$, and $K$ the trace field of $M$. Clearly we have $K \subset k$. Now $M$ being singular, it contains a sharp hypersurface $N$ in its boundary, whose field of definition must be $k$ by Corollary 2.3. Using Remark 1.3 it is easily checked that the field of definition of $N$ (seen as a $(n-1)$-manifold) corresponds to the trace field of $\pi_{1}(N)$, seen as a subgroup of $\mathrm{PO}(n, 1)$. We conclude that $k \subset K$, and thus $M$ and $M_{0}$ (resp. their fundamental groups $\Gamma$ and $\Gamma_{0}$ ) have the same trace field. Let $\mathbf{G}$ be the ambient group of $\Gamma_{0}$. From the inclusion $\Gamma \subset \Gamma_{0}$ we have $\Gamma \subset \mathbf{G}(k)$, and it follows that $\mathbf{G}$ is the ambient group of $\Gamma$ as well (by uniqueness).

We will say that an arithmetic piece $M$ (resp. its fundamental group) is defined over $k$ if its trace field is $k$; this extends the terminology used for regular pieces (see Remark 1.3).

Corollary 2.6. For $i=0,1$ let $M_{i}$ be an arithmetic piece containing a hypersurface $N_{i}$ in its boundary. If $N_{0}$ is isometric to $N_{1}$ then $M_{0}$ and $M_{1}$ are defined over the same field.

Proof. Being isometric we have that $N_{0}$ and $N_{1}$ have the same field of definition, and the result follows immediately from Corollary 2.3 and Lemma 2.5 .

2.6. Gluings. Let $M$ be a hyperbolic manifold obtained by gluing arithmetic pieces. By definition this means that there exists a sequence

$$
M_{1}, \ldots, M_{r}=M
$$


where $M_{1}$ is an arithmetic piece, and for $i \geq 1$ the piece $M_{i+1}$ is obtained from $M_{i}$ by one of the two following operations:

(I) gluing an arithmetic piece $M_{0}$ to $M_{i}$ using an isometry $\phi$ between two hypersurfaces in their respective boundary, i.e.,

$$
M_{i+1}=M_{0} \cup_{\phi} M_{i} .
$$

(II) "closing up" two hypersurfaces in the boundary of $M_{i}$, i.e., $M_{i+1}=$ $M_{i} /\{x=\phi(x)\}$ where $\phi$ is an isometry between two hypersurfaces in the boundary of $M_{i}$.

Theorem 1.5 is a specialization of the following.

Theorem 2.7. Let $M$ be a hyperbolic piece of dimension $n>3$ obtained by gluing a finite number of arithmetic pieces $M_{i}$ defined over $k$.

(1) The trace field of $M$ is a totally real multiquadratic extension $K / k$.

(2) The ambient group of $M$ is $\mathbf{G}_{K}$, where $\mathbf{G}$ is the ambient group of any of the piece $M_{i}$.

Proof. We proceed inductively. If $M$ is an arithmetic piece over $k$ then (1) and (2) hold trivially. Suppose then that $M_{1}$ is a piece constructed by gluing arithmetic pieces over $k$, and that it respects the conditions (1) and (2) (recurrence assumption). We will glue $M_{1}$ using a sharp hypersurface $N_{1} \subset M_{1}$. Let $K_{1}$ be the trace field of $M_{1}$, which is multiquadratic over $k$. Let $\mathbf{G}=\mathbf{P O}^{+}(V, f)$ be the ambient group of an arithmetic piece containing $N_{1}$, so that $\mathbf{G}_{K_{1}}$ is the ambient group of $M_{1}$. Thus we may write $M_{1}=\Gamma_{1} \backslash \widetilde{M}_{1}$, for $\widetilde{M}_{1} \subset \mathbf{H}(V, f)$ and $\Gamma_{1}$ a discrete subgroup of $\mathbf{G}\left(K_{1}\right)$ stabilizing $\widetilde{M}$.

Let us first examine the case of the gluing of type (I), i.e., $M$ is obtained by gluing $M_{1}$ to an arithmetic piece $M_{0}$ along a hypersurface $N_{0} \subset M_{0}$ isometric to $N_{1}$. Abstractly we have $\pi_{1}(M)=\left\langle\pi_{1}\left(M_{0}\right), \pi_{1}\left(M_{1}\right)\right\rangle$. By Corollary 2.6 we have that $M_{0}$ is defined over $k$. Moreover, it is of the first type (Prop. 2.2), and we can write $M_{0}=\Gamma_{0} \backslash \widetilde{M}_{0}$, where $\widetilde{M}_{0} \subset \mathbf{H}\left(V_{0}, f_{0}\right)$ for some admissible quadratic space $\left(V_{0}, f_{0}\right)$ over $k$, and $\Gamma_{0} \subset \mathbf{P} \mathbf{O}_{f_{0}}^{+}(k)$. We fix a hyperplane lift $\mathbf{H}\left(W_{0}\right)$ of $N_{0}$ in $\mathbf{H}\left(V_{0}, f_{0}\right)$, which is necessarily $k$-rational by Prop. 2.2. We fix as well a hyperplane lift $\mathbf{H}\left(W_{1}\right) \subset$ $\mathbf{H}(V, f)$ of $N_{1}$; it is $k$-rational for the same reason. Since $N_{1}$ and $N_{0}$ are isometric, the quadratic spaces $W_{1}$ and $W_{0}$ must be similar over $k$ (see [10, 2.6]). More precisely, there exists a similitude $\varphi: W_{0} \rightarrow W_{1}$ whose extension to $W_{0} \otimes_{k} \mathbb{R}$ lifts the gluing isometry between $N_{0}$ and $N_{1}$. By Lemma 2.4 there exists an extension $F / k$, at most quadratic, over which $\varphi$ extends to a similitude $\phi: V_{0} \otimes_{k} F \rightarrow V \otimes_{k} F$ (and note that the scaling factor can be chosen totally positive here, so that $F$ is totally real). In particular, $\operatorname{conj}(\phi)\left(\Gamma_{0}\right) \subset \mathbf{G}(F)$. The map $\phi$ induces an isometry between $\mathbf{H}\left(V_{0}, f_{0}\right)$ and $\mathbf{H}(V, f)$ that matches the lift hyperplanes of $N_{0}$ and $N_{1}$. Possibly after composing with the reflection through $\mathbf{H}\left(W_{1}\right)$ (which is $k$-rational by Lemma 2.1), we can hence see the respective universal covers $\widetilde{M}_{0}$ and $\widetilde{M}_{1}$ of $M_{0}$ and $M_{1}$ as lying on both sides of $\mathbf{H}(W)$ in $\mathbf{H}(V, f)$. We can thus write $M=\Gamma \backslash \mathbf{H}(V, f)$, where $\Gamma=\left\langle\operatorname{conj}(\phi)\left(\Gamma_{0}\right), \Gamma_{1}\right\rangle$. Hence $\Gamma \subset \mathbf{G}\left(F_{1}\right)$, where $F_{1}$ is the composite of $F$ and $K_{1}$. In particular, the trace field $K$ of $\Gamma$ is either $K_{1}$ or $F_{1}$, which in either case is totally real and multiquadratic over $k$. It follows that $\mathbf{G}_{K}$ is the ambient group of $\Gamma$.

Finally note that the inductive proof can be started with any arithmetic piece. Thus the group $\mathbf{G}$ can be the ambient group of any of these pieces, as stated in (2). 
For a gluing of type (II), we consider two isometric sharp hypersurfaces $N_{0}, N_{1} \subset$ $M_{1}$. If $M_{0} \subset M_{1}$ is an arithmetic piece containing $N_{0}$ and $\left(V_{0}, f_{0}\right)$ and $W_{0}$ are defined as above, the same argument gives a similitude $\phi: V_{0} \otimes_{k} F \rightarrow V \otimes_{k} F$, where $F / k$ is a totally real extension which is at most quadratic. Now by the induction hypothesis, $\mathbf{P O}^{+}\left(V_{0}, f_{0}\right)_{K_{1}}$ is an ambient group for $\Gamma_{1}$. It follows that we have an isometry $\psi: \mathbf{H}\left(V_{0}, f_{0}\right) \rightarrow \mathbf{H}(V, f)$ such that $\operatorname{conj}(\psi)$ is defined over $K_{1}$ and $g=\phi \circ \psi^{-1}$ (seen as an element of $\mathbf{G}(\mathbb{R})$ ) lifts an isometry $N_{1} \cong N_{0}$. Now by construction, there exists $g^{\prime} \in \mathbf{G}(K)$ such that $\Gamma=\left\langle\Gamma_{1}, g^{\prime}\right\rangle$, and $g^{\prime}$ induces the isometry $N_{1} \cong N_{0}$. It follows that $g$ and $g^{\prime}$ coincide on $\mathbf{H}\left(W_{1}\right)$, and thus $g \in\left\{g^{\prime}, g^{\prime} \rho\right\}$ where $\rho$ denotes reflection through $\mathbf{H}\left(W_{1}\right)$. As before, this shows that $\Gamma \subset \mathbf{G}\left(F_{1}\right)$, where $F_{1}$ is the composite of $F$ and $K_{1}$. Thus the trace field $K$ of $\Gamma$ is multiquadratic over $k$, and $\mathbf{G}_{K}$ is the ambient group of $\Gamma$.

\section{Homology AND VOLUmes}

The main result of this section is Theorem 3.5 of which Theorem 1.12 will be a direct consequence. Since these results hold up to commensurability, we will restrict ourselves to considering lattices that are torsion-free and orientation-preserving. The basic idea is, given an ambient group $\mathbf{G}_{K}$, to compare fundamental classes of lattices $\Gamma \subset \mathbf{G}(K)$ in a $\mathbb{Q}$-vector space $\mathcal{L}(\mathbf{G})$; the latter will be defined in Sect. 3.1 . Many of the proofs are generalizations of the arguments in [7], which deals with quasi-arithmetic lattices. Prop. 3.4 is new; it is the key result needed at the level of ambient groups. Its proof is given in Sect. 3.4

3.1. Fundamental classes and volumes. Let $G=\operatorname{PO}(n, 1)$ and let $\Omega=\partial \mathbf{H}^{n}$ denote the geometric boundary of $\mathbf{H}^{n}$. Any subgroup $S \subset G$ acts naturally on $\Omega$; we write $H_{n}(S, \Omega)$ for the homology of $S$ relative to $\Omega$; see [7, Sect. 1.6]. By definition $H_{n}(S, \Omega)$ is the homology group $H_{n-1}(S ; J \Omega)$ with coefficients in the kernel $J \Omega$ of the augmentation map $\mathbb{Z} \Omega \rightarrow \mathbb{Z}$, and it follows that there is a canonical "connecting" map from the usual homology:

$$
\delta: H_{n}(S) \rightarrow H_{n}(S, \Omega) .
$$

If $S$ acts freely on $\Omega$, this map is an isomorphism.

In the case where $S=\Gamma \subset G^{\circ}$ is a torsion-free lattice, we have that $H_{n}(\Gamma, \Omega)$ is isomorphic to the singular homology of the end-compactification $\widehat{M}$ of $M=$ $\Gamma \backslash \mathbf{H}^{n}$ (see [7, Sect. 3]). Thus $H_{n}(\Gamma, \Omega) \cong \mathbb{Z}$; let $\xi$ denote the generator that corresponds to the positive orientation in $H_{n}(\widehat{M})$. We define the fundamental class $[\Gamma] \in H_{n}(G, \Omega ; \mathbb{Q})$ as the image of $\xi$ under the map induced by the inclusion $\Gamma \subset G$. This notion captures the volume:

Proposition 3.1. There exists a linear map $v: H_{n}(G, \Omega ; \mathbb{Q}) \rightarrow \mathbb{R}$ such that $v([\Gamma])=$ $\operatorname{vol}\left(\Gamma \backslash \mathbf{H}^{n}\right)$ for any torsion-free lattice $\Gamma \subset G^{\circ}$.

Proof. See [7, Prop. 1.7].

3.2. The space $\mathcal{L}(\mathbf{G})$. Let $K \subset \mathbb{R}$ be a number field, and let $\mathbf{G}$ be a connected $K$-group such that $\mathbf{G}(\mathbb{R})^{\circ}=\mathrm{PO}(n, 1)^{\circ}$; for the moment we are not assuming that $\mathbf{G}$ is admissible (nor pseudo-admissible). The inclusion $\mathbf{G}(K) \subset \mathbf{G}(\mathbb{R})$ composed with the map $\delta$ in (3.1) induces a map

$$
H_{n}(\mathbf{G}(K) ; \mathbb{Q}) \rightarrow H_{n}(\mathbf{G}(\mathbb{R}), \Omega ; \mathbb{Q}),
$$


whose image will be denoted by $\mathcal{L}(\mathbf{G})$. If $\mathbf{G}=\mathbf{G}_{0, K}$ for some $k$-group $\mathbf{G}_{0}$ and some extension $K / k$ (so that $\mathbf{G}_{0}(\mathbb{R})=\mathbf{G}(\mathbb{R})$ ), then $\mathcal{L}\left(\mathbf{G}_{0}\right) \subset \mathcal{L}(\mathbf{G})$ (where $\mathcal{L}\left(\mathbf{G}_{0}\right)$ is the image of $\left.H_{n}\left(\mathbf{G}_{0}(k) ; \mathbb{Q}\right)\right)$.

Lemma 3.2. Let $\Lambda \subset \mathbf{G}(K) \cap \mathbf{G}(\mathbb{R})^{\circ}$ be a torsion-free lattice. Then $[\Lambda]$ lies in $\mathcal{L}(\mathbf{G})$.

Proof. The exact sequence $0 \rightarrow J \Omega \rightarrow \mathbb{Z} \Omega \rightarrow \mathbb{Z} \rightarrow 0$ together with the inclusion $\Lambda \subset \mathbf{G}(K)$ give rise to the following diagram with exact rows, which appears in [7, Eq. (6.2)]:

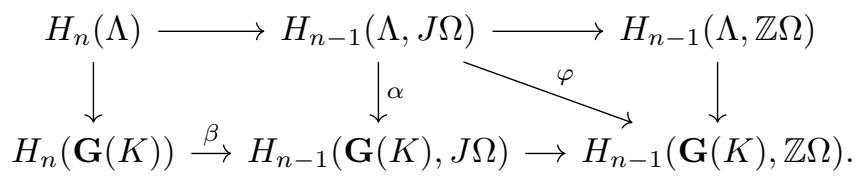

By definition, $[\Lambda]$ is the image under $\alpha$ of a generator $\xi$ of $H_{n-1}(\Lambda, J \Omega)=H_{n}(\Lambda, \Omega) \cong$ $\mathbb{Z}$. By Prop. 6.1 of [7] (whose proof works in our more general setting), there is an integer $N$ such that $\varphi(N \cdot \xi)=0$, and thus when tensoring with $\mathbb{Q}$ we get $\varphi(\xi)=0$. Hence over $\mathbb{Q}$, we have that $\alpha(\xi)=[\Lambda]$ is in the image of $\beta$ and therefore also in $\mathcal{L}(\mathbf{G})$.

This last result permits to view the fundamental class of a lattice $\Gamma$ as an element of $\mathcal{L}(\mathbf{G})$, where $\mathbf{G}$ is the ambient group of $\Gamma$. By Prop. 3.1 the fundamental class $[\Gamma]$ determines the volume; the space $\mathcal{L}(\mathbf{G})$ can be thought as a replacement for the Bloch group, which appears in the case of dimension $n=3$ (see for instance [17, Sect. 12.7]). In the rest of this section we will show that if $\Gamma$ is pseudo-arithmetic of the first type, one can exhibit generators for $\mathcal{L}(\mathbf{G})$ corresponding to fundamental classes of arithmetic lattices.

Remark 3.3. The definition of $\mathcal{L}(\mathbf{G})$ is comparable to the "higher groups" $\mathscr{P}_{n}$ in Neumann and Yang [22, Sect. 8], with the difference (crucial for our applications) that we work with $K$-points instead of $\mathbb{R}$-points.

3.3. Subordinated fundamental classes. Assume now that $\mathbf{G}=\mathbf{P O}_{f, K}^{+}$is pseudo-admissible over $K / k$ with $f$ a quadratic form over $k$. As in Sect. 1.6 we write $f=f\left(x_{0}, \ldots, x_{n}\right)$ in a diagonal form with negative $x_{0}$ coefficient, and $K=k\left(\sqrt{a_{1}}, \ldots, \sqrt{a_{r}}\right)$ with $a_{j} \in k$ totally positive. For $i=\left(i_{1}, \ldots, i_{r}\right) \in\{0,1\}^{r}$ we set $\alpha_{i}=\sqrt{a_{1} i_{1} \cdots a_{r}{ }^{i_{r}}}$. Then the $\alpha_{i}$ form a basis of $K / k$.

We let $\mathbf{G}_{i}$ denote the $k$-group $\mathbf{P} \mathbf{O}_{f_{i}}^{+}$, where $f_{i}=f\left(x_{0}, \ldots, x_{n-1}, \alpha_{i} x_{n}\right)$. The diagonal matrix $D_{i}$ with entries $1, \ldots, 1, \alpha_{i}$ satisfies $f_{i}=f \circ D_{i}$, and induces (via conjugation) an isomorphism of algebraic groups $\mathbf{G}_{i, K} \stackrel{\sim}{\rightarrow} \mathbf{G}$. In particular, it induces an inclusion $\mathbf{G}_{i}(k) \hookrightarrow \mathbf{G}(K)$. The proof of the following proposition is the subject of Sect. 3.4.

Proposition 3.4. The following natural map, induced by the inclusions $\mathbf{G}_{i}(k) \hookrightarrow$ $\mathbf{G}(K)$, is surjective:

$$
\bigoplus_{i \in\{0,1\}^{r}} H_{n}\left(\mathbf{G}_{i}(k) ; \mathbb{Q}\right) \longrightarrow H_{n}(\mathbf{G}(K) ; \mathbb{Q}) .
$$

Assuming the proposition, we are ready to state and prove the main theorem of this section. Together with Prop. 3.1 it directly implies Theorem 1.12 
Theorem 3.5. Let $\mathbf{G}=\mathbf{P O}_{f, K}^{+}$be as above. For each $i \in\{0,1\}^{r}$, let $\Gamma_{i}$ be a torsion-free arithmetic lattice in $\mathbf{G}_{i}(k) \cap G^{\circ}$. Then the $\mathbb{Q}$-vector space $\mathcal{L}(\mathbf{G})$ is generated by the set of fundamental classes $\left\{\left[\Gamma_{i}\right] \mid i \in\{0,1\}^{r}\right\}$.

Proof. The algebraic groups $\mathbf{G}_{i}$ are admissible over $k$, and therefore [7, Prop. 4.2] shows that $H_{n}(\mathbf{G}(k) ; \mathbb{Q})$ has dimension one. The fact that the volume of $\Gamma_{i} \backslash \mathbf{H}^{n}$ is non-zero combined with Prop. 3.1 and Lemma 3.2 implies that $\mathcal{L}\left(\mathbf{G}_{i}\right)$ is of dimension one and generated by $\left[\Gamma_{i}\right]$. Now Prop. 3.4 shows that $\mathcal{L}(\mathbf{G})$ is generated by its subspaces $\mathcal{L}\left(\mathbf{G}_{i}\right)$, and this finishes the proof.

Remark 3.6. We do not claim that the classes $\left[\Gamma_{i}\right]$ are linearly independent. For $n$ odd it might well be the case, since there are no expected congruences for the special values of $L$-functions that appears in the covolumes. But this justification certainly fails for $n$ even, and it is not excluded that $\mathcal{L}(\mathbf{G})$ has dimension 1 there.

3.4. The homology of ambient groups. The goal of this section is to prove Prop. 3.4. We begin by relating the homology of an algebraic group to that of its simply-connected cover.

Lemma 3.7. Let $\mathbf{G}$ be a connected simple adjoint algebraic group defined over a field $K$, and let $\widetilde{\mathbf{G}}$ denote its simply-connected cover. The natural map $\widetilde{\mathbf{G}}(K) \rightarrow$ $\mathbf{G}(K)$ induces a surjective map

$$
H_{n}(\widetilde{\mathbf{G}}(K) ; \mathbb{Q}) \rightarrow H_{n}(\mathbf{G}(K) ; \mathbb{Q}) .
$$

Proof. We borrow the notation from the proof of [7, Prop. 4.2], since our proof is essentially the same. In particular, let $C$ denote the center of $\widetilde{\mathbf{G}}$. Consider the following composition of maps

$$
\begin{aligned}
H_{n}(\widetilde{\mathbf{G}}(K) ; \mathbb{Q}) \longrightarrow & H_{n}(\widetilde{\mathbf{G}}(K) / C(K) ; \mathbb{Q}) \\
& \downarrow \\
& H_{n}(\widetilde{\mathbf{G}}(K) / C(K) ; \mathbb{Q})_{A} \longrightarrow H_{n}(\mathbf{G}(K) ; \mathbb{Q})
\end{aligned}
$$

where the horizontal maps are induced by the quotient $\widetilde{\mathbf{G}}(K) \rightarrow \widetilde{\mathbf{G}}(K) / C(K)$ and the inclusion $\widetilde{\mathbf{G}}(K) / C(K) \hookrightarrow \mathbf{G}(K)$ coming from the exact sequence (4.3) in [7], respectively.

These horizontal maps coincide with the edge homomorphisms of the spectral sequences (4.5) and (4.4) in [7] respectively, which are isomorphisms since both sequences collapse at $E^{2}$ (see [33, 6.8.2]). The vertical map is obviously surjective, and looking at the definition of edge homomorphisms it is routine to check that the composition of the three maps is induced by $\widetilde{\mathbf{G}}(K) \rightarrow \mathbf{G}(K)$.

Proof of Proposition 3.4. Let $\lambda_{i}$ be the inclusion $\mathbf{G}_{i}(k) \rightarrow \mathbf{G}(K)$ defined in Sect. 3.3. Since $\lambda_{i}$ is defined by conjugating the quadratic form $f$, we may lift it at the level of the Spin groups, i.e., at the level of the simply-connected covers of $\mathbf{G}_{i}$ and $\mathbf{G}$. Now using Lemma 3.7, we see that to prove Prop. 3.4 it suffices to show that the induced map

$$
\bigoplus_{i \in\{0,1\}^{r}} H_{n}\left(\widetilde{\mathbf{G}}_{i}(k) ; \mathbb{Q}\right) \longrightarrow H_{n}(\widetilde{\mathbf{G}}(K) ; \mathbb{Q})
$$

is surjective. We will show that it is actually an isomorphism. 
Recall (see [3, Sect. 6.3] and [7, Sect. 4]) that for the cohomology with coefficients in $\mathbb{R}$ we have two canonical isomorphisms from the continuous cohomology of $\widetilde{\mathbf{G}}_{i}(k \otimes \mathbb{Q})$ :

$$
H^{n}\left(\widetilde{\mathbf{G}}_{i}(k) ; \mathbb{R}\right) \stackrel{\sim}{\leftarrow} H_{\mathrm{ct}}^{n}\left(\widetilde{\mathbf{G}}_{i}(k \otimes \mathbb{Q} \mathbb{R}) ; \mathbb{R}\right) \stackrel{\sim}{\rightarrow} H^{n}\left(X_{i} ; \mathbb{R}\right),
$$

where $X_{i}$ is the compact dual space (or compact twin) associated with $\widetilde{\mathbf{G}}_{i}\left(k \otimes_{\mathbb{Q}} \mathbb{R}\right)$. For $\mathbf{G}_{i}$ admissible, $X_{i}$ is the $n$-sphere; more precisely, $X_{i}$ is the symmetric space associated with $\hat{f}_{i}$, the positive definite version of $f_{i}$ (i.e., $f_{i}$ with the coefficient of $x_{0}$ made positive). It follows that the left hand side of (3.3) has dimension $s=2^{r}$. The same argument shows that the dimension of $H^{n}(\widetilde{\mathbf{G}}(K) ; \mathbb{R})$ equals that of $H^{n}(X ; \mathbb{R})$, where the compact dual $X$ associated with $\widetilde{\mathbf{G}}\left(K \otimes_{\mathbb{Q}} \mathbb{R}\right)$ is a product $S^{n} \times \cdots \times S^{n}$ of $s$ copies of the $n$-sphere; this dimension is $s$ by the Künneth formula.

To prove that (3.3) is indeed an isomorphism it clearly suffices to show that the same map with coefficients extended to $\mathbb{R}$ is an isomorphism. This allows (after dualizing the cohomology) to use the canonical maps in (3.4) and their analogues for $\widetilde{\mathbf{G}}$. Observe that the first map in (3.4) is induced by the inclusion $k \subset k \otimes_{\mathbb{Q}} \mathbb{R}$ and the second is just the natural isomorphism between the corresponding Lie algebras (combined with multiplication by a scalar, see [3. Sect. 6.3]). Both maps are functorial in the sense that they commute with the maps induced by the inclusions $\mathbf{G}_{i}(k) \rightarrow \mathbf{G}(K)$. Then it is enough to show that the map

$$
\theta: \bigoplus_{i \in\{0,1\}^{r}} H_{n}\left(X_{i} ; \mathbb{R}\right) \longrightarrow H_{n}\left(S^{n} \times \cdots \times S^{n} ; \mathbb{R}\right)
$$

is an isomorphism. For the multi-index $0=(0, \ldots, 0)$ we fix a generator $\left[X_{0}\right]$ of $H_{n}\left(X_{0} ; \mathbb{R}\right)$. In (3.4) the only contribution to the cohomology of $\widetilde{\mathbf{G}}_{0}\left(k \otimes_{\mathbb{Q}} \mathbb{R}\right)$ comes from the noncompact factor $\widetilde{\mathbf{G}}_{0}(\mathbb{R})$. Similarly for $\widetilde{\mathbf{G}}\left(K \otimes_{\mathbb{Q}} \mathbb{R}\right)$, whose noncompact factors form a product $\prod_{\sigma} \widetilde{\mathbf{G}}_{0}(\mathbb{R})$ indexed by the set of embeddings $\sigma: K \rightarrow \mathbb{R}$ fixing $k$ (since $\widetilde{\mathbf{G}}=\widetilde{\mathbf{G}}_{0, K}$ ), that is, by the Galois group $\operatorname{Gal}(K / k)$ since $K / k$ is Galois. The Künneth formula then gives an isomorphism

$$
H_{n}\left(S^{n} \times \cdots \times S^{n} ; \mathbb{R}\right) \cong \bigoplus_{\sigma} H_{n}\left(S^{n} ; \mathbb{R}\right),
$$

with the right hand side indexed by $\operatorname{Gal}(K / k)$. Let $\left(\left[S_{\sigma}\right]\right)_{\sigma}$ denote the image of $\left[X_{0}\right]$ via (3.5).

The matrix $D_{i}=\operatorname{diag}\left(1, \ldots, 1, \alpha_{i}\right)$ induces a homeomorphism $X_{i} \cong X_{0}$ (since $\left.\hat{f}_{0} \circ D_{i}=\hat{f}_{i}\right)$ and thus an isomorphism in homology; we denote by $\left[X_{i}\right] \in H_{n}\left(X_{i} ; \mathbb{R}\right)$ the image of $\left[X_{0}\right]$. A careful analysis of the maps $\lambda_{i}$ then shows that $\theta\left(\left[X_{i}\right]\right)=$ $\left(\left(\sigma\left(\alpha_{i}\right) / \alpha_{i}\right)\left[S_{\sigma}\right]\right)_{\sigma}$. With respect to the bases $\left\{\left[X_{i}\right]\right\}$ and $\left\{\left[S_{\sigma}\right]\right\}$ the map $\theta$ is thus given by the matrix $B=\left(\sigma\left(\alpha_{i}\right) / \alpha_{i}\right)$. Its determinant equals

$$
\operatorname{det}(B)=\prod_{i} \alpha_{i}^{-1} \cdot \Delta\left[\left\{\alpha_{i}\right\}\right],
$$

where $\Delta\left[\left\{\alpha_{i}\right\}\right]$ is the discriminant in $K / k$ of the basis $\left\{\alpha_{i}\right\}$. Hence $\operatorname{det}(B)$ is nonzero, and the proposition is proved.

\section{REFLECTION GROUPS AND PSEUDO-ADMISSIBILITY}

In this section we explain how to check if a given hyperbolic reflection group is pseudo-arithmetic. We have applied the method to all examples contained in the 
tables provided by the program CoxIter developed by Guglielmetti [11]. We have found that all these reflection groups are pseudo-arithmetic for $n>3$; see Sect. 4.3 for a summary of the results.

4.1. Ambient groups of Coxeter groups. The references for this section are Vinberg [31, Theorem 5] and Maclachlan [16, Sect. 9]. Let $q=-x_{0}^{2}+x_{1}^{2}+\cdots+x_{n}^{2}$ denote the standard quadratic form in $\mathbf{H}^{n}$, with associated bilinear form $(x, y) \mapsto$ $B(x, y)=q(x+y)-q(x)-q(y)$. Let $P \subset \mathbf{H}^{n}$ be a Coxeter polytope with $r$ faces whose orthogonal vectors are denoted by $e_{1}, \ldots, e_{r}$, and are chosen to have $q$-norm one. Let $\Gamma \subset \mathrm{PO}(n, 1)$ be the Coxeter group associated with $P$, and let $G=\left(a_{i j}\right)_{i j}$ be its Gram matrix; its entries are $a_{i j}=B\left(e_{i}, e_{j}\right)$. For a subset $\left\{i_{1}, \ldots, i_{k}\right\} \subset\{1, \ldots, r\}$, define

$$
b_{i_{1}, \ldots, i_{k}}=a_{i_{1} i_{2}} \cdots a_{i_{k-1} i_{k}} a_{i_{k} i_{1}} \quad \text { and } \quad v_{i_{1}, \ldots, i_{k}}=\left(a_{1 i_{1}} a_{i_{1} i_{2}} \cdots a_{i_{k-1} i_{k}}\right) e_{i_{k}} .
$$

The trace field of $\Gamma$ is then the field $K=\mathbb{Q}\left(\left\{b_{i_{1}}, \ldots, i_{k}\right\}\right)$ generated by all the possible "cyclic" products $b_{i_{1}, \ldots, i_{k}}$, and the ambient group is the group $\mathbf{P} \mathbf{O}_{f}$ where $f$ is the quadratic form $q$ restricted to the $K$-vector space $V$ spanned by the $v_{i_{1}, \ldots, i_{k}}$. Note that the quadratic form $f$ can be determined without explicitly computing the $e_{i}$; one proceeds as follows:

(1) Extract a minor $M$ of the Gram matrix which has full rank $n+1$. This minor is then given by $M=\left(B\left(e_{m_{i}}, e_{m_{j}}\right)\right)_{i j}$ for some sequence $m_{1}, \ldots, m_{n+1}$, and it follows that the vectors $e_{m_{1}}, \ldots e_{m_{n+1}}$ are linearly independent over $\mathbb{R}$.

(2) For each $j=1, \ldots, n+1$, find a sequence of indices $l_{1}, \ldots, l_{k}$ with $l_{k}=m_{j}$ such that the product $a_{1 l_{1}} a_{l_{1} l_{2}} \cdots a_{l_{k-1} l_{k}}$ is non-zero (possible since the Coxeter diagram is connected), and set $w_{j}=v_{l_{1}, \ldots, l_{k}}$.

(3) The $K$-vector space $V$ then admits $\left\{w_{1}, \ldots, w_{n+1}\right\}$ as a basis. It follows that $f$ has the matrix representation $A=\left(B\left(w_{i}, w_{j}\right)\right)_{i j}$, whose entries can be easily computed from the entries of $G$.

4.2. Quadratic forms over multiquadratic extensions. For this section we refer to Lam's book 15 and the two papers 5, 6, of Elman, Lam and Wadsworth. If $k$ is a field, $W(k)$ will denote the Witt group (or ring) of $k$ (as defined in [15, Chap. II]). Recall that two rank $n$ regular quadratic forms $f, f^{\prime}$ over $k$ are isometric if and only if they represent the same element in $W(k)$, and that $0 \in W(k)$ is represented by any hyperbolic form. Since we will use this group only to compare regular forms of the same rank, we will usually identify quadratic forms with their image in $W(k)$.

For a field extension $K / k$, we let $r_{k}^{K}: W(k) \rightarrow W(K)$ denote the group homomorphism induced by the inclusion $k \subset K$ (sending a quadratic form $f$ to $f_{K}$, the same form seen over $K$ ). If $K / k$ is a quadratic extension with $K=k(\sqrt{a}), a \in k$, let $s: K \rightarrow k$ be the $k$-linear map sending $1 \mapsto 0$ and $\sqrt{a} \mapsto 1$. This map induces a group homomorphism $s_{k}^{K}: W(K) \rightarrow W(k)$ called the transfer map: a quadratic form $q \in W(K)$ of rank $n$ is mapped to $s_{k}^{K}(q)=s \circ q$, seen as a quadratic form of rank $2 n$ over $k$. See [15, Sect. VII.3] for details.

Let now $K / k$ be a multiquadratic extension of a global field $k$. The two results we will need for our criterion are:

(1) $K / k$ is an excellent extension, i.e., a quadratic form $q$ is defined over $k$ (by which we mean $q \cong f_{K}$ for some quadratic form $f$ over $k$ ) if and only if $q \in \operatorname{im}\left(r_{k}^{K}\right)$. This is a consequence of [5, Theorem 2.13] which states that 
TABLE 1. Coxeter groups in 28, with trace field of degree 8 and 4.

\begin{tabular}{|c|c|c|c|c|}
\hline $\operatorname{deg}$ & $\begin{array}{l}\text { Reference } \\
{[28 \text {, page(item)] }}\end{array}$ & $\operatorname{dim}$ & Trace field & $\begin{array}{l}\text { Quadratic form } \\
f=f_{0}+a x_{n}^{2}\end{array}$ \\
\hline 8 & $\begin{array}{l}9(\mathrm{e}) \\
11(\mathrm{e})\end{array}$ & 4 & $\mathbb{Q}(\sqrt{2}, \sqrt{3}, \sqrt{5})$ & $a=1$ \\
\hline \multirow{11}{*}{4} & \multirow{6}{*}{$\begin{array}{l}9(\mathrm{a}) \\
9(\mathrm{c}) \\
9(\mathrm{~d}) \\
9(\mathrm{f}) \\
11(\mathrm{a}) \\
11(\mathrm{c}) \\
11(\mathrm{~d}) \\
11(\mathrm{f})\end{array}$} & \multirow{6}{*}{4} & $\mathbb{Q}(\sqrt{2}, \sqrt{15})$ & \multirow{4}{*}{$\begin{array}{l}a=3 \\
a=2 \\
a=1 \\
a=2\end{array}$} \\
\hline & & & & \\
\hline & & & $\mathbb{Q}(\sqrt{3}, \sqrt{5})$ & \\
\hline & & & & \\
\hline & & & $\mathbb{Q}(\sqrt{2}, \sqrt{3})$ & \multirow[b]{2}{*}{$a=1$} \\
\hline & & & $\mathbb{Q}(\sqrt{5}, \sqrt{6})$ & \\
\hline & \multirow{4}{*}{$\begin{array}{l}14(\mathrm{c}) \\
17(\mathrm{~d}) \\
14(\mathrm{~d}) \\
17(\mathrm{c}) \\
19(\mathrm{e})\end{array}$} & \multirow{4}{*}{5} & & \multirow{4}{*}{$a=1$} \\
\hline & & & $\mathbb{Q}(\sqrt{ } 2, \sqrt{ } 3)$ & \\
\hline & & & $\mathbb{Q}(\sqrt{2}, \sqrt{5})$ & \\
\hline & & & $\mathbb{Q}(\sqrt{2}, \sqrt{13})$ & \\
\hline & $\begin{array}{l}23(\mathrm{e}) \\
24(\mathrm{~h})\end{array}$ & 6 & $\mathbb{Q}(\sqrt{2}, \sqrt{3})$ & $a=1$ \\
\hline
\end{tabular}

any extension of a global field $k$ containing a Galois extension of $k$ of even degree is excellent. See also [15, Sect. XII.4].

(2) There is an exact sequence of Witt groups

$$
W(k) \stackrel{r_{k}^{K}}{\longrightarrow} W(K) \stackrel{\prod s_{F}^{K}}{\longrightarrow} \prod W(F),
$$

where the product on the right is taken on all fields $k \subset F \subset K$ with $[K: F]=2$. This is the end of the sequence (1.1) in [6], whose exactness is proven for global fields in Theorem $\mathrm{C}$ of the same paper.

From these two results we deduce:

Proposition 4.1. A regular quadratic form $q \in W(K)$ is defined over $k$ if and only if for all subfields $k \subset F \subset K$ with $[K: F]=2$, we have $s_{F}^{K}(q)=0 \in W(F)$, that is, if the corresponding form is hyperbolic.

4.3. The computations. Let $\Gamma \subset \mathrm{PO}(n, 1)$ be a Coxeter group with trace field $K$ and ambient group $\mathbf{P O}_{f}$ for some quadratic form $f$ over $K$. In order to check if $\Gamma$ is pseudo-admissible, one needs to find a subfield $k \subset K$ such that $K / k$ is multiquadratic and a $k$-form of $\mathbf{P O} \mathbf{O}_{f}$ which is admissible. Using Prop. 4.1, one can already find a candidate for $k$ : the intersection of all codimension 2 subfields $F \subset K$ such that $s_{F}^{K}(f)$ is hyperbolic. If one is then able to find an admissible quadratic form $g$ over $k$ such that $f \cong g_{K}$, one can conclude: $\Gamma$ is pseudo-arithmetic over $K / k$ with ambient group $\mathbf{P O}_{g}$.

If however the field $K$ is multiquadratic over $\mathbb{Q}$ and the form $f$ is itself defined over $\mathbb{Q}$, the computation of such a $g$ becomes superfluous, since any quadratic form over $\mathbb{Q}$ of signature $(n, 1)$ is admissible. It turns out that all the examples in the 
TABle 2. Coxeter groups in 28] with trace field of degree 2.

\begin{tabular}{|c|c|c|c|c|}
\hline $\operatorname{deg}$ & Reference [28, page(item)] & $\operatorname{dim}$ & Trace field & $\begin{array}{l}\text { Quadratic form } \\
f=f_{0}+a x_{n}^{2}\end{array}$ \\
\hline \multirow{28}{*}{2} & $08(\mathrm{~d}), 08(\mathrm{e})$ & & $\mathbb{Q}(\sqrt{15})$ & $a=6$ \\
\hline & $08(\mathrm{~b}), 12(\mathrm{~b})$ & & $\mathbb{Q}(\sqrt{3})$ & $a=1$ \\
\hline & $10(d), 11(b)$ & 4 & $\mathbb{Q}(\sqrt{3})$ & $a=2$ \\
\hline & $08(\mathrm{f})$ & 4 & $\mathbb{Q}(\sqrt{30})$ & $a=3$ \\
\hline & $09(\mathrm{~b})$ & & $\mathbb{Q}(\sqrt{30})$ & $a=6$ \\
\hline & $10(\mathrm{~b}), 10(\mathrm{c})$ & & $\mathbb{Q}(\sqrt{6})$ & $a=1$ \\
\hline & $13(\mathrm{~d}), 13(\mathrm{f}), 16(\mathrm{~d}), 16(\mathrm{f})$ & \multirow{8}{*}{5} & $\mathbb{Q}(\sqrt{10})$ & $a=2$ \\
\hline & $19(\mathrm{~d}), 19(\mathrm{f})$ & & $\mathbb{Q}(\sqrt{13})$ & $a=1$ \\
\hline & 18(b), 18(c), 18(e), 20(b), & & $\mathbb{Q}(\sqrt{2})$ & $a=1$ \\
\hline & $\begin{array}{l}20(\mathrm{c}), 20(\mathrm{e}), 21(\mathrm{~b}), 21(\mathrm{~d}), \\
22(\mathrm{a})\end{array}$ & & & \\
\hline & $19(\mathrm{~b}), 19(\mathrm{c})$ & & $\mathbb{Q}(\sqrt{26})$ & $a=2$ \\
\hline & $14(\mathrm{a}), 14(\mathrm{e}), 17(\mathrm{~b}), 17(\mathrm{f})$ & & $\mathbb{Q}(\sqrt{3})$ & $a=1$ \\
\hline & $\begin{array}{l}\text { 14(b), 14(f), 15(c), 15(e), } \\
17(\mathrm{a}), 17(\mathrm{e})\end{array}$ & & $\mathbb{Q}(\sqrt{5})$ & $a=1$ \\
\hline & $13(\mathrm{c}), 13(\mathrm{e}), 16(\mathrm{c}), 16(\mathrm{e})$ & & $\mathbb{Q}(\sqrt{6})$ & $a=2$ \\
\hline & $26(\mathrm{~d}), 26(\mathrm{e})$ & \multirow{6}{*}{6} & $\mathbb{Q}(\sqrt{10})$ & $a=1$ \\
\hline & $24(\mathrm{~b}), 24(\mathrm{c}), 25(\mathrm{c}), 25(\mathrm{~d})$ & & $\mathbb{Q}(\sqrt{2})$ & $a=1$ \\
\hline & $25(\mathrm{f}), 25(\mathrm{~g})$ & & & \\
\hline & $23(\mathrm{~d}), 23(\mathrm{f}), 24(\mathrm{~g}), 25(\mathrm{a})$ & & $\mathbb{Q}(\sqrt{3})$ & $a=1$ \\
\hline & $26(\mathrm{a}), 26(\mathrm{~b})$ & & $\mathbb{Q}(\sqrt{35})$ & $a=10$ \\
\hline & $23(\mathrm{~b}), 23(\mathrm{c}), 24(\mathrm{e}), 24(\mathrm{f})$ & & $\mathbb{Q}(\sqrt{6})$ & $a=2$ \\
\hline & $27(\mathrm{~b}), 27(\mathrm{c})$ & \multirow{4}{*}{7} & $\mathbb{Q}(\sqrt{22})$ & $a=2$ \\
\hline & $27(\mathrm{e}), 27(\mathrm{f})$ & & $\mathbb{Q}(\sqrt{3})$ & $a=1$ \\
\hline & $28(\mathrm{e}), 28(\mathrm{f})$ & & $\mathbb{Q}(\sqrt{5})$ & $a=3$ \\
\hline & $28(\mathrm{~b}), 28(\mathrm{c}), 29(\mathrm{~b}), 29(\mathrm{c})$ & & $\mathbb{Q}(\sqrt{6})$ & $a=2$ \\
\hline & $30(\mathrm{f}), 31(\mathrm{a})$ & \multirow{2}{*}{8} & $\mathbb{Q}(\sqrt{3})$ & $a=2$ \\
\hline & $30(b), 30(c)$ & & $\mathbb{Q}(\sqrt{5})$ & $a=2$ \\
\hline & $32(\mathrm{~b}), 32(\mathrm{c})$ & 9 & $\mathbb{Q}(\sqrt{5})$ & $a=1$ \\
\hline & $33(\mathrm{~b}), 33(\mathrm{c})$ & 10 & $\mathbb{Q}(\sqrt{2})$ & $a=1$ \\
\hline
\end{tabular}

table of Coxeter groups of [1] fulfill this simpler necessary condition for pseudoarithmeticity.

The computations were done using the mathematical program Sage 29 and an algorithm of [14] for checking hyperbolicity over number fields. In Tables 12 we show a sample of the Coxeter groups we analyzed, namely those from the classification of Roberts 28] having trace field of degree $>1$. Each of these Coxeter groups is pseudo-arithmetic over $K / \mathbb{Q}$ (where $K$ is the trace field). The indicated quadratic forms were found by brute-force search over quadratic forms of the shape $f_{0}+a x_{n}^{2}$, where $f_{0}=-x_{0}^{2}+x_{1}^{2}+\cdots+x_{n-1}^{2}$. 


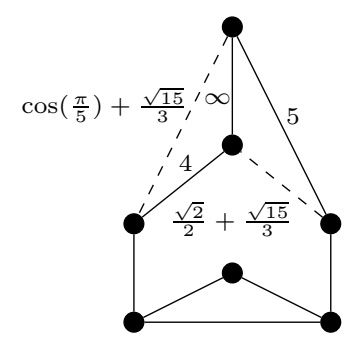

(1) $[28$, p. 9 (e)]

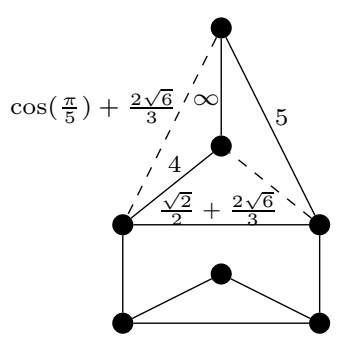

(2) [28, p. $11(\mathrm{e})]$

Figure 2. Two examples of Coxeter polytopes in $\mathbf{H}^{4}$ with trace field of degree 8 over $\mathbb{Q}$.

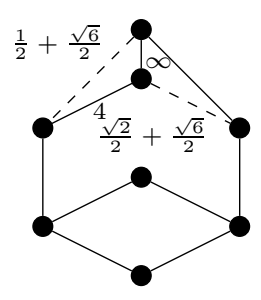

(1) 28, p. $14(\mathrm{c})$

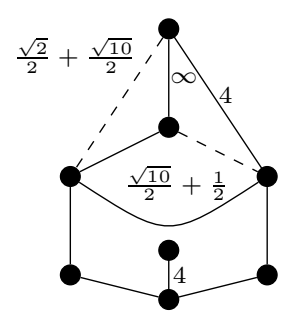

(3) 28, p. 17 (c)]

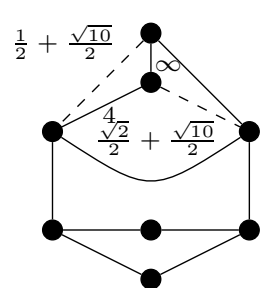

(2) [28, p. $14(\mathrm{~d})]$

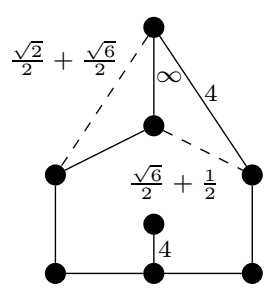

(4) [28, p. $17(\mathrm{~d})]$

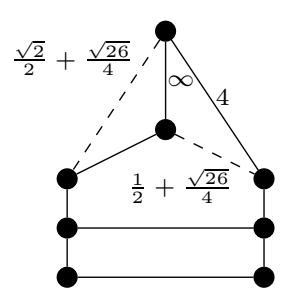

(5) 28 , p. $19(\mathrm{e})]$

FiguRE 3. Five examples of Coxeter polytopes in $\mathbf{H}^{5}$ with trace field of degree 4 over $\mathbb{Q}$.

Finally, in Figures 2 and 3 we reproduce the diagrams of the Coxeter groups realizing the largest trace fields in even dimensions $(n=4$ : degree 8$)$ and odd dimensions ( $n=5$ : degree 4$)$. 


\section{ACKNOWLEDGMENTS}

We would like to thank Ruth Kellerhals and Steve Tschantz for their help concerning Example1.13 Andrei Rapinchuk for pointing out the result of Lemma 1.11, Martin Deraux for his explanation concerning Sect. 1.7.1, and Matthew Stover and Jean Raimbault for helpful discussions. We also thank the referee for helpful comments.

\section{REFERENCES}

[1] Mikhail Belolipetsky, Arithmetic hyperbolic reflection groups, Bull. Amer. Math. Soc. (N.S.) 53 (2016), no. 3, 437-475, DOI 10.1090/bull/1530. MR 3501796

[2] Mikhail V. Belolipetsky and Scott A. Thomson, Systoles of hyperbolic manifolds, Algebr. Geom. Topol. 11 (2011), no. 3, 1455-1469, DOI 10.2140/agt.2011.11.1455. MR.2821431

[3] José I. Burgos Gil, The regulators of Beilinson and Borel, CRM Monograph Series, vol. 15, American Mathematical Society, Providence, RI, 2002. MR.1869655

[4] Martin Deraux, John R. Parker, and Julien Paupert, New non-arithmetic complex hyperbolic lattices, Invent. Math. 203 (2016), no. 3, 681-771, DOI 10.1007/s00222-015-0600-1. MR.3461365

[5] Richard Elman, T. Y. Lam, and Adrian R. Wadsworth, Amenable fields and Pfister extensions, Conference on Quadratic Forms-1976 (Proc. Conf., Queen's Univ., Kingston, Ont., 1976), Queen's Papers in Pure and Appl. Math., No. 46, Queen's Univ., Kingston, Ont., 1977, pp. 445-492. With an appendix "Excellence of $F(\varphi) / F$ for 2-fold Pfister forms" by J. K. Arason. MR0560497

[6] Richard Elman, T. Y. Lam, and Adrian R. Wadsworth, Quadratic forms under multiquadratic extensions, Nederl. Akad. Wetensch. Indag. Math. 42 (1980), no. 2, 131-145. MR577569

[7] Vincent Emery, On volumes of quasi-arithmetic hyperbolic lattices, Selecta Math. (N.S.) 23 (2017), no. 4, 2849-2862, DOI 10.1007/s00029-017-0308-8. MR3703466

[8] David Fisher, Jean-François Lafont, Nicholas Miller, and Matthew Stover, Finiteness of maximal geodesic submanifolds in hyperbolic hybrids, J. Eur. Math. Soc. (JEMS), to appear.

[9] Tsachik Gelander and Arie Levit, Counting commensurability classes of hyperbolic manifolds, Geom. Funct. Anal. 24 (2014), no. 5, 1431-1447, DOI 10.1007/s00039-014-0294-3. MR.3261631

[10] M. Gromov and I. Piatetski-Shapiro, Nonarithmetic groups in Lobachevsky spaces, Inst. Hautes Études Sci. Publ. Math. 66 (1988), 93-103. MR932135

[11] R. Guglielmetti, CoxIter-computing invariants of hyperbolic Coxeter groups, LMS J. Comput. Math. 18 (2015), no. 1, 754-773, DOI 10.1112/S1461157015000273. MR3434903

[12] N. W. Johnson, J. G. Ratcliffe, R. Kellerhals, and S. T. Tschantz, The size of a hyperbolic Coxeter simplex, Transform. Groups 4 (1999), no. 4, 329-353, DOI 10.1007/BF01238563. MR.1726696

[13] Max-Albert Knus, Alexander Merkurjev, Markus Rost, and Jean-Pierre Tignol, The book of involutions, American Mathematical Society Colloquium Publications, vol. 44, American Mathematical Society, Providence, RI, 1998. With a preface in French by J. Tits. MR 1632779

[14] Przemysław Koprowski and Alfred Czogała, Computing with quadratic forms over number fields, J. Symbolic Comput. 89 (2018), 129-145, DOI 10.1016/j.jsc.2017.11.009. MR3804811

[15] T. Y. Lam, Introduction to quadratic forms over fields, Graduate Studies in Mathematics, vol. 67, American Mathematical Society, Providence, RI, 2005. MR2104929

[16] Colin Maclachlan, Commensurability classes of discrete arithmetic hyperbolic groups, Groups Geom. Dyn. 5 (2011), no. 4, 767-785, DOI 10.4171/GGD/147. MR2836459

[17] Colin Maclachlan and Alan W. Reid, The arithmetic of hyperbolic 3-manifolds, Graduate Texts in Mathematics, vol. 219, Springer-Verlag, New York, 2003. MR1937957

[18] Bruno Martelli, An introduction to geometric topology, CreateSpace Independent Publishing Platform, 2016.

[19] Jeffrey S. Meyer, Totally geodesic spectra of arithmetic hyperbolic spaces, Trans. Amer. Math. Soc. 369 (2017), no. 11, 7549-7588, DOI 10.1090/tran/6970. MR.3695838

[20] Olivier Mila, The trace field of hyperbolic gluings, arXiv:1911.13157 2019.

[21] Olivier Mila, The trace field of hyperbolic gluings, Ph.D. thesis, Universität Bern, 2019. 
[22] Walter D. Neumann and Jun Yang, Bloch invariants of hyperbolic 3-manifolds, Duke Math. J. 96 (1999), no. 1, 29-59, DOI 10.1215/S0012-7094-99-09602-3. MR.1663915

[23] E. B. Vinberg, V. V. Gorbatsevich, and O. V. Shvartsman, Discrete subgroups of Lie groups / MR0968445 (90c:22036)], Lie groups and Lie algebras II, Encyclopaedia Math. Sci., vol. 21, Springer, Berlin, 2000, pp. 1-123, 217-223. MR1756407

[24] Takashi Ono, On algebraic groups and discontinuous groups, Nagoya Math. J. 27 (1966), 279-322. MR.199193

[25] Gopal Prasad, Volumes of S-arithmetic quotients of semi-simple groups, Inst. Hautes Études Sci. Publ. Math. 69 (1989), 91-117. With an appendix by Moshe Jarden and the author. MR.1019962

[26] Gopal Prasad and Andrei S. Rapinchuk, Weakly commensurable arithmetic groups and isospectral locally symmetric spaces, Publ. Math. Inst. Hautes Études Sci. 109 (2009), 113184, DOI 10.1007/s10240-009-0019-6. MR2511587

[27] Jean Raimbault, A note on maximal lattice growth in $\mathrm{SO}(1, n)$, Int. Math. Res. Not. IMRN 16 (2013), 3722-3731, DOI 10.1093/imrn/rns151. MR3090707

[28] Mike Roberts, A classification of non-compact Coxeter polytopes with $n+3$ facets and one non-simple vertex, preprint arXiv:1511.08451, 2015.

[29] The Sage Developers, Sagemath, the Sage Mathematics Software System (Version 7.6), 2017, http://www.sagemath.org.

[30] È. B. Vinberg, Discrete groups generated by reflections in Lobačevskǐ spaces (Russian), Mat. Sb. (N.S.) 72 (114) (1967), 471-488; correction, ibid. 73 (115) (1967), 303. MR0207853

[31] È. B. Vinberg, Rings of definition of dense subgroups of semisimple linear groups, Math. USSR Izvestija 5 (1971), no. 1, 45-55.

[32] E. B. Vinberg, Non-arithmetic hyperbolic reflection groups in higher dimensions (English, with English and Russian summaries), Mosc. Math. J. 15 (2015), no. 3, 593-602, 606, DOI 10.17323/1609-4514-2015-15-3-593-602. MR3427439

[33] Charles A. Weibel, An introduction to homological algebra, Cambridge Studies in Advanced Mathematics, vol. 38, Cambridge University Press, Cambridge, 1994. MR 1269324

[34] D. B. Zagier, Zetafunktionen und quadratische Körper (German), Springer-Verlag, BerlinNew York, 1981. Eine Einführung in die höhere Zahlentheorie. [An introduction to higher number theory]; Hochschultext. [University Text]. MR631688

Mathematisches Institut, Universität Bern, Sidlerstrasse 5, CH-3012 Bern, SwitzerLAND

Email address: vincent.emery@math.ch

Centre de recherches mathématiques, Université de Montréal, Pavillon AndréAisenstadt, Montréal, Québec, H3T 1J4, Canada

Email address: olivier.mila@umontreal.ca 